[8] G. Pfurtscheller, C. Neuper, G. Müller, B. Obermaier, G. Krausz, A. Schlögl, R. Scherer, B. Graimann, C. Keinrath, D. Skliris, M. Woertz, G. Supp, and C. Schrank, "Graz BCI: State of the art and clinical applications," IEEE Trans. Neural Syst. Rehabil. Eng., vol. 11, no. 2, pp. 177-180, Jun. 2003.

[9] K. R. Müller, C. W. Anderson, and G. E. Birch, "Linear and nonlinear methods for brain-computer interfaces," IEEE Trans. Neural Syst. Rehabil. Eng., vol. 11, no. 2, pp. 165-169, Jun. 2003.

[10] N. Birbaumer, T. Hinterberger, A. Kübler, and N. Neumann, "The thought-translation device (TTD): Neurobehavioral mechanisms and clinical outcome," IEEE Trans. Neural Syst. Rehabil. Eng., vol. 11, no. 2, pp. 120-123, Jun. 2003.

[11] J. del R. Millán, "Brain-computer interfaces," in The Handbook of Brain Theory and Neural Networks, M. A. Arbib, Ed., 2nd ed. Cambridge, MA: MIT Press, 2002, pp. 178-181.

[12] G. Pfurtscheller, C. Neuper, C. Guger, W. Harkam, H. Ramoser, A. Schlögl, B. Obermaier, and M. Pregenzer, "Current trends in graz brain-computer interface (BCI) research," IEEE Trans. Rehabil. Eng., vol. 8, no. 2, pp. 216-219, Jun. 2000.

[13] D. J. McFarland, A. T. Lefkowicz, and J. R. Wolpaw, "Design and operation of an EEG-based brain-computer interface with digital signal processing technology," Behavior Res. Meth., Instrum., Comput., vol. 29, pp. 337-345, 1997.

[14] J. del R. Millán, "On the need for on-line learning in brain-computer interfaces," presented at the Int. Joint Conf. Neural Networks, Budapest, Hungary, 2004.

[15] T. Nykopp, J. Heikkonen, and M. Sams, "Sequential classification of finger movements from MEG recordings," in Biomedisinische Technik, Proc. 2nd Int. Brain-Computer Interface Workshop and Training Course (Graz), 2004, pp. 77-78.

[16] A. Buttfield, P. W. Ferrez, and J. del R. Millán, "TTowards a robust BCI: error potentials and online learning," IEEE Trans. Neural Syst. Rehabil. Eng., vol. 14, no. 2, pp. 164-168, Jun. 2006.

[17] P. Sykacek, S. J. Roberts, and M. Stokes, "Adaptive BCI based on variational bayesian kalman filtering: An empirical evaluation," IEEE. Trans. Biomed. Eng., vol. 51, no. 5, pp. 719-727, May 2004.

[18] M. Kraudelat, P. Shenoy, B. Blankertz, R. P. N. Rao, and K.-R. Müller, "Online adaptation for CSP based BCI systems," presented at the Brain-Computer Interface Technology: 3rd Int. Meeting, Rensselaerville, NY, Jun. 14-19, 2005.

[19] A. Schlög1, C. Vidaurre, and G. Pfurtscheller, "Assessing nonstationarities in BCI data," presented at the Brain-Computer Interface Technology: 3rd Int. Meeting, Rensselaerville, NY, Jun. 14-19, 2005.

[20] C. Neuper, A. Schlögl, and G. Pfurtscheller, "Enhancement of left-right sensorimotor EEG differences during feedback-regulated motor imagery," J. Clin. Neurophysiol., vol. 16, pp. 373-382, 2000.

[21] A. Schlögl, D. Flotzinger, and G. Pfurtscheller, "Adaptive autoregressive modeling used for single-trial EEG classification," Biomed. Technik, vol. 42, pp. 162-167, 1997.

[22] A. Schlögl, The Electroencephalogram and the Adaptive Autoregressive Model: Theory and Applications. Aachen, Germany: Shaker Verlag, 2000.

[23] G. Pfurtscheller, C. Neuper, A. Schlögl, and K. Lugger, "Separability of EEG signals recorded during right and left motor imagery using adaptive autoregressive parameters," IEEE Trans. Rehabil. Eng., vol. 6, no. 3, pp. 316-325, Sep. 1998.

[24] C. Guger, A. Schlögl, C. Neuper, C. Walterspacher, D. Strein, T. Pfurtscheller, and G. Pfurtscheller, "Rapid prototyping of an EEG-based brain-computer interface (BCI)," IEEE Trans. Neural Syst. Rehabil. Eng., vol. 9, no. 1, pp. 49—58, Mar. 2001.

[25] G. Pfurtscheller, C. Neuper, D. Flotzinger, and M. Pregenzer, "EEG-based discrimination between imagination of right and left hand movement," Electroenceph. Clin. Neurophysiol., vol. 103, pp. 642-651, 1997.

[26] C. Vidaurre, A. Schlögl, R. Cabeza, R. Scherer, and G. Pfurtscheller, "A fully on-line adaptive BCI," IEEE Trans. Biomed. Eng., vol. 53, no. 6, pp. 1214-1219, Jun. 2006.

[27] S. Haykin, Adaptive Filter Theory. Upper Saddle River, NJ: Prentice Hall, 1996.

[28] A. Schlögl, C. Neuper, and G. Pfurtscheller, "Estimating the mutual information of an EEG-based brain-computer interface," Biomed. Technik, vol. 47, pp. 3-8, 2002.
[29] G. Krausz, R. Scherer, G. Korisek, and G. Pfurtscheller, "Critical decision-speed and information transfer in the graz brain-computer-interface," Appl. Psychophysiol. Biofeedback, vol. 28, pp. 233-240, Sep. 2003.

[30] G. Pfurtscheller and C. Neuper, "Motor imagery and direct brain-computer communications," Proc. IEEE, vol. 89, no. 7, pp. 1123-1134, Jul. 2001.

[31] — , "Motor imagery activates primary sensorimotor area in humans," Neurosci. Lett., vol. 239, pp. 65-68, 1997.

[32] C. Vidaurre, "On-line adaptive classification for brain-computer interfaces," Ph.D. dissertation, Public Univ. Navarre, Navarre, Spain, 2006.

\section{Estimation of the Uncertainty in Time Domain Indices of RR Time Series}

\author{
Miguel A. García-González*, Mireya Fernández-Chimeno, and
} J. Ramos-Castro

\begin{abstract}
A method for estimating the uncertainty in time-domain indices of RR time series is described. The method relies on the central limit theorem that states that the distribution of a sample average of independent samples has an uncertainty that asymptotically approaches to the sample standard deviation divided by the square root of the number of samples. Because RR time series cannot be characterized by a set of independent samples, we propose to estimate the uncertainty of indices by computing them in blocks that satisfy that the obtained partial indices are independent. We propose a methodology to search sets of independent partial indices and apply this methodology to the estimation of the uncertainty in the mean RR, SDRR, and r-msDD indices. The results show that the uncertainty can be higher than the $10 \%$ of the index for the SDRR and even higher for the r-msDD. Moreover, a statistical test for the difference of two indices is proposed.
\end{abstract}

\section{INTRODUCTION}

Heart rate variability (HRV) is regarded as a marker of the relationship between the cardiovascular and autonomic nervous systems (see the Task Force of the European Society of Cardiology and the North American Society of Pacing and Electrophysiology [1], for a good review on HRV applications and recommendations for measurements). There are several techniques that quantify the beat-to-beat fluctuations although, nowadays, the time-domain analysis is the most employed methodology in clinical studies, both in short-term and 24-h recordings.

Besides of applications of time domain analysis of HRV based on 24-h recordings, such as prediction of cardiac disease and mortality [2]-[4], HRV analysis from short-term recordings is becoming a powerful tool for the prediction of several diseases (mortality prediction after myocardial infarction [5], studies on diabetic patients [6], assessment of training status in athletes [7], or assessment of psychological stress [8]). Among the whole proposed time-domain indices, the most recurrently calculated are the mean $\left(\mathrm{RR}_{\text {mean }}\right)$, the standard deviation

Manuscript received November 14, 2005; revised July 22, 2006. Asterisk indicates corresponding author

*M. García-González is with the Research Centre on Biomedical Engineering (CREB), Department of Electronic Engineering (DEE), Universitat Politècnica de Catalunya (UPC), C/ Jordi Girona 1-3, Edifici C-4, 08034 Barcelona, Spain (e-mail: mireya@eel.upc.edu).

M. Fernández-Chimeno and J. Ramos-Castro are with the Research Centre on Biomedical Engineering (CREB), Department of Electronic Engineering (DEE), Universitat Politècnica de Catalunya (UPC), C/ Jordi Girona 1-3, 08034 Barcelona, Spain.

Digital Object Identifier 10.1109/TBME.2006.890513 
(SDRR), and the standard deviation of the differentiation (r-msDD) of the RR time series. The aim of this paper is to provide a methodology to estimate the uncertainty in the quantification of these three indices from a statistical point of view. For each index obtained from a single RR time series, the method must provide a number for the quantification of the distrust on the result of the index (the uncertainty of the index), and an interval centered on the obtained index that contains the "true" value of the index with a certain probability [the confidence interval $(\mathrm{CI})]$.

When characterizing a RR time series, the number of available RR samples $(N)$ is always finite and, very often, limited. When computing an index from the $N$ intervals we estimate a characteristic of the time series (i.e., the sample mean of the RR samples is an estimation of the expectation of the time series). If the RR time series is stationary and other new $N$ intervals were obtained, the new estimation of the index will be different although similar. The estimation of uncertainty quantifies this variation.

In Sections II-IV, an introduction to the estimation of the uncertainty is provided and the proposed methodology is outlined. The methodology is characterized by comparison with Monte Carlo simulations by employing artificial time series and, finally, the method is applied to real RR time series.

\section{MAterials AND MethodS}

\section{A. Estimation and Uncertainty}

From the Central Limit Theorem, the arithmetic mean $\left(O_{\text {mean }}\right)$ of $M$ independent observations $\left(\left\{o_{1}, o_{2}, \ldots o_{j}, \ldots, o_{M}\right\}\right)$ is, when $M$ sufficiently high, a random variable with normal distribution whose standard deviation can be estimated as

$$
\begin{aligned}
u\left(O_{\text {mean }}\right) & =s_{O_{\text {mean }}}=\frac{s_{O}}{\sqrt{M}} \\
& =\frac{\sqrt{\frac{1}{M-1} \sum_{i=1}^{M}\left(o_{i}-\frac{1}{M} \cdot \sum_{i=1}^{M} o_{i}\right)^{2}}}{\sqrt{M}} .
\end{aligned}
$$

The Guide for the Expression of the Uncertainty in Measurement [9] defines the standard deviation of the mean $\left(u\left(O_{\text {mean }}\right)\right)$ as the type-A standard uncertainty. Regardless the number of available observations, a CI can be defined as

$$
\begin{aligned}
\operatorname{Prob}\left\{O_{\text {mean }}-k \cdot u\left(O_{\text {mean }}\right) \leq\right. & \bar{O} \\
& \left.\leq O_{\text {mean }}+k \cdot u\left(O_{\text {mean }}\right)\right\}=p
\end{aligned}
$$

where $k$ is the coverage factor and can be obtained from the t-Student distribution tables with $M-1$ degrees of freedom and $p$ is the coverage probability (the probability that the true value would be inside the specified $\mathrm{CI}$ ) and $\bar{O}$ is the mean (expectation) of the population. The product $k \cdot u\left(O_{\text {mean }}\right)$ is defined as the type-A expanded uncertainty $\left(U\left(O_{\text {mean }}\right)\right)$. Note that these calculations are true regardless the distribution of the observations provided that the number of observations is high enough and that the observations are independent. This approach can not be applied directly to the estimation of the uncertainty of the mean of RR time series because the samples are seldom independent.

Let us suppose that we want to estimate the uncertainty of an index from a time series with $N$ nonindependent samples. If the index can be estimated as an average of partial indices computed in $M$ nonoverlapped blocks of size $n(n \cdot M \leq N)$, the standard uncertainty in the index can be estimated as

$$
u(I) \approx \frac{s_{p I}}{\sqrt{M}}=\sqrt{\frac{\sum_{j=1}^{M}\left(p I_{j}-\frac{1}{M} \sum_{i=1}^{M} p I_{i}\right)^{2}}{M \cdot(M-1)}}
$$

provided that the partial indices $\left(p I_{i}, i \in[1, M]\right)$ are independent. Accordingly, a CI can be obtained. This approximation holds if the RR time series can be considered as a stationary process (if not, in fact, the mean is not strictly defined although, of course, the sample mean can be computed) and because the sample mean is an unbiased estimator of the expectation.

There is a tradeoff between $n$ and $M$ If $n$ is high, the knowledge of each $p I_{i}$ is higher but the results of a test of independence can be compromised because $M$ is low. If $M$ is high, the estimation of each $p I_{i}$ can be meaningless. As explained above, the different $p I_{i}$ must be independent. In this paper, we have considered that they are independent if the run test and the reverse arrangement test with a confidence level of the $95 \%$ indicate that the independence hypothesis can be accepted. Bendat and Piersol, [10] provide a detailed description of both tests. Next, there is a brief summary of these tests.

\section{B. Independence Tests}

The independence tests are based on the assumption that independent data have no trend. Bendat and Piersol [10] propose two nonparametric procedures to find if the data are independent or not: the Run Test (RT) that can detect fluctuating sets and the Reverse Arrangement Test (RAT) that is more sensitive to monotonic trends. Let us consider that we have $M$ partial indices and want to test if they are independent.

In the RT, the sample mean of the partial indices is calculated and removed from each partial index. Then, the sign sequence is created

$$
d p I_{i}=p I_{i}-\frac{1}{M} \cdot \sum_{j=1}^{M} p I_{j} \quad \operatorname{sig}(i)=\left\{\begin{array}{ll}
1, & \text { if } d p I_{i} \geq 0 \\
-1, & \text { if } d p I_{i}<0
\end{array} .\right.
$$

A run is defined as a portion of the sign sequence delimited by sign changes. For example, if $\operatorname{sig}(i)=\{1,1,1,-1,-1,1,-1,1\}$ we have $r=5$ runs in 8 observations. A fitting of the lower $(L r)$ and upper $(U r)$ limits for a confidence of $95 \%$ is $(M<100)$

$$
\begin{aligned}
L r & =0.45 \cdot M-2.85 \\
U r & =0.55 \cdot M+3.85 .
\end{aligned}
$$

If $r$ is outside the interval $(L r, U r)$ it can be concluded that the partial indices are nonindependent.

In the RAT, the following matrix is created

$$
h_{i j}=\left\{\begin{array}{ll}
1, & \text { if } p I_{i}>p I_{j} \text { and } i<j \\
0, & \text { otherwhise }
\end{array} .\right.
$$

And, the following parameter is computed:

$$
A=\sum_{i=1}^{M-1}\left(\sum_{j=i+1}^{M} h_{i j}\right)
$$




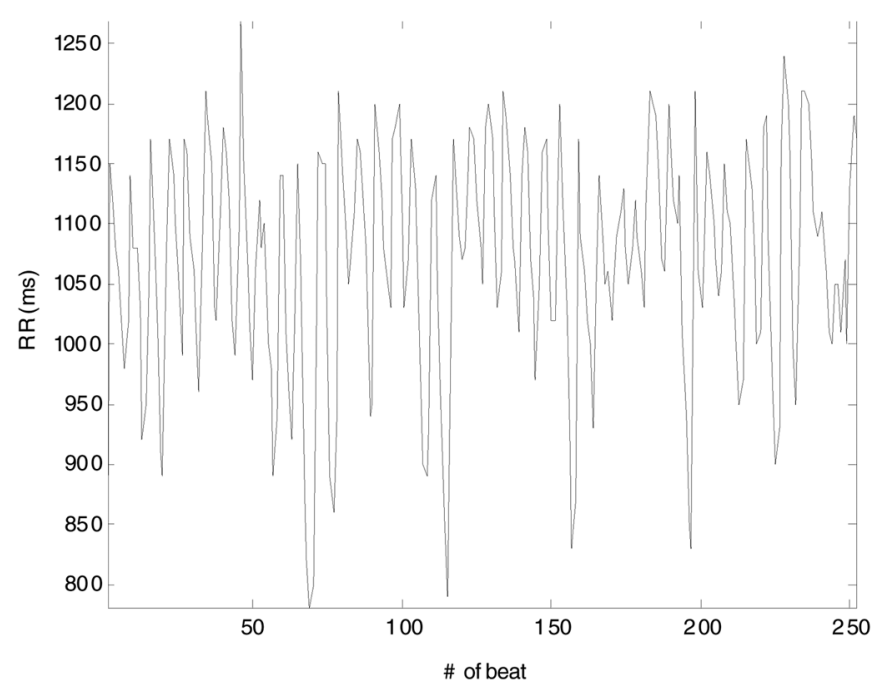

Fig. 1. RR time series with 252 samples used to show the behavior of independent partial indices.

TABLE I

RESULTS FOR THE INDEPENDENCE TESTS FOR THE EXAMPLE OF A RR TIME SERIES. IN THE RAW DATA SECTION, THE INDEPENDENCE OF THE RR SAMPLES ARE TESTED WhILE IN THE PARTIAL INDICES SECTION, THE INDEPENDENCE OF $M=16$ PARTIAL STANDARD DEVIATIONS IS TESTED

\begin{tabular}{|l|l|l|l|l|}
\hline Test & \multicolumn{2}{|l}{} & \multicolumn{2}{l|}{ RT } \\
\hline \multirow{5}{*}{ Raw data } & $\mathrm{r}$ & 78 & $A$ & 14272 \\
\cline { 2 - 5 } & $L r$ & 111.1 & $L A$ & 16567 \\
\cline { 2 - 5 } & $U r$ & 141.9 & $U A$ & 17739 \\
\cline { 2 - 5 } & Comparison & $\mathrm{r}<L r<U r$ & Comparison & $A<L A<U A$ \\
\cline { 2 - 5 } & Independent? & $\mathrm{NO}$ & Independent? & NO \\
\hline \multirow{4}{*}{$\begin{array}{l}\text { Partial indices } \\
(M=16)\end{array}$} & $\mathrm{r}$ & 5 & $A$ & 76 \\
\cline { 2 - 5 } & $L r$ & 4.4 & $L A$ & 40.4 \\
\cline { 2 - 5 } & $U r$ & 12.6 & $U A$ & 77.5 \\
\cline { 2 - 5 } & Comparison & $L r<\mathrm{r}<U r$ & Comparison & $L A<A<U A$ \\
\cline { 2 - 5 } & Independent? & YES & Independent? & YES \\
\hline
\end{tabular}

In this test, the $95 \% \mathrm{CI}$ can be fitted as

$$
\begin{aligned}
L A & =0.089 \cdot M^{2.21} \\
U A & =0.352 \cdot M^{1.95}
\end{aligned}
$$

As an example of the technique, Fig. 1 shows a real RR time series with 252 intervals. The RT and RAT on the raw RR time series indicate that the intervals are not independent as shown in Table I. With $M=16$, both tests indicate that the standard deviation computed in the blocks can be considered as independent. Based on the independence of the obtained standard deviations, an estimation of the uncertainty of the SDRR will be $6.4 \mathrm{~ms}$ (the calculated SDRR is $95.7 \mathrm{~ms}$ ) because the standard deviation of the calculated standard deviations is $25.4 \mathrm{~ms}$ and $25.4 \mathrm{~ms} /(16)^{0.5} \cong 6.4 \mathrm{~ms}$. The $6.4 \mathrm{~ms}$ result is for this particular partition of the time series. However, several partitions of the time series may provide independent partial indices and different results. Let us see how to evaluate these differences.

\section{Strategy for the Choice of an Independent Set of Partial Indices}

As seen in the prior example, the obtained set of partial standard deviations was classified as independent although $r$ was very close to $L r$ and $A$ was very close to $U A$. Maybe another combination of $M$ and starting interval (the first block started in the first RR sample) would provide values of $r$ and $A$ closer to the midpoint of each CI. Let us define the set of possible $M$ and the set of possible starting RR samples as

$$
\begin{aligned}
& M \in\left\{10,\left\lfloor\frac{N}{10}\right\rfloor\right\} \\
& i_{o} \in\left\{1, N-\left\lfloor\frac{N}{M}\right\rfloor \cdot M+1\right\}
\end{aligned}
$$

where \lfloor\rfloor is the rounding to the lowest integer operator.

In the example of Fig. 1, $M$ can adopt every integer value between 10 and 25 . If $M=15$, there are 13 possible starting RR samples $\left(i_{o}\right)$. The blocks are defined as

$$
\begin{aligned}
& \mathbf{R R}_{i}^{b}=\left\{\operatorname{RR}\left(i_{o}+(i-1) \cdot\left\lfloor\frac{N}{M}\right\rfloor\right),\right. \\
& \operatorname{RR}\left(i_{o}+1+(i-1) \cdot\left\lfloor\frac{N}{M}\right\rfloor\right), \ldots, \\
&\left.\operatorname{RR}\left(i_{o}+\left\lfloor\frac{N}{M}\right\rfloor-1+(i-1) \cdot\left\lfloor\frac{N}{M}\right\rfloor\right)\right\} \\
& i \in\{1, M\} .
\end{aligned}
$$

In the example there are 134 possible combinations of $M$ and $i_{o}$. The strategy is to compute all the available blocks and to provide an independence factor (IF) that can guide us to the determination of what is the best combination of $M$ and $i_{o}$. Let us define the IF as

$$
\mathrm{IF}=\sqrt{\left(\frac{\left|\frac{U r+L r}{2}-r\right|}{\frac{U r-L r}{2}}\right)^{2}+\left(\frac{\left|\frac{U A+L A}{2}-A\right|}{\frac{U A-L A}{2}}\right)^{2}} .
$$

The independence factor will be zero if both tests have the discriminating parameter just in the centre of the CI. In this paper, we have tried two methods in order to provide a representative value of the uncertainty of the index.

- Method of the minimum (M1): From all the obtained sets, find the one that has the minimum IF. The standard uncertainty in the index is obtained with (3) for the chosen set of $p I_{i}$. In the case of the time series of Fig. 1, the minimum IF is 0,047 and corresponds to a partition in 11 blocks starting with the first RR sample. In this case, the uncertainty of the standard deviation computed with (3) is $6.30 \mathrm{~ms}$.

- Method of the first quartile (M2): Identify how many sets have IF $<1$. From this reduced number of sets, identify which ones have an IF inside the first quartile. Once identified, express the standard uncertainty of the index as the mean of the standard uncertainties obtained on the sets inside the first quartile. Fig. 2 illustrates the procedure for the time series of Fig. 1 when measuring the uncertainty in SDRR resulting an uncertainty of $6.01 \mathrm{~ms}$. If in this method there are no sets with IF $<1$ then M1 is applied.

\section{Intervals of Confidence}

The asymptotic distribution of the mean of partial indices is normal because they are independent. For each set, a CI can be obtained by finding the coverage factor in a t-Student table (the number of degrees of freedom is $M-1$ ). When employing M1, the interval of confidence will be calculated by this direct form. Nevertheless, when using M2, an average of uncertainties is provided. Which coverage factor has to be employed in this case? As recommended in the Guide, when using a confidence level of $95 \%$, the coverage factor will be 2 and for a confidence level of $99 \%$, the coverage factor will be 3 . Returning to the 


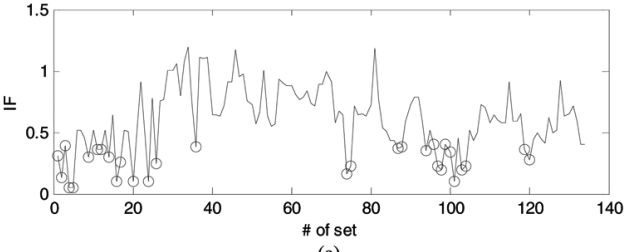

(a)

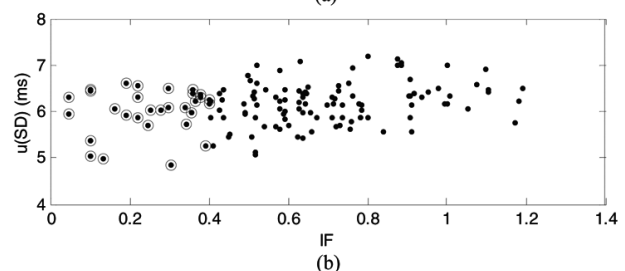

(b)

Fig. 2. Procedure to obtain independent partial indices by election of $M$ and $i_{o}$. (a) Independence factor (IF) for all possible sets (134) obtained from the RR time series of Fig. 1. The minimum IF is 0.047 so this set is the one considered in M1. The circles represent the IF values of the first quartile. (b) Uncertainty of the SDRR computed for every IF. The circles also show the cases of IF in the first quartile.

example of Fig. 1, M1 establishes an expanded uncertainty (95\% confidence) of $U(\mathrm{SDRR})_{M 1}=2.23 \cdot 6.30 \mathrm{~ms} \cong 14.0 \mathrm{~ms}$ while M2 establishes $U(\mathrm{SDRR})_{M 2}=2 \cdot 6.01 \mathrm{~ms} \cong 12.0 \mathrm{~ms}$. In order to discern which procedure is more accurate, simulation studies must be carried out.

\section{E. Characterization and Application of the Methods}

1) White Uniform Noise: M1 and M2 have been applied to the determination of the uncertainty of $\mathrm{RR}_{\text {mean }}$, SDRR, and r-msDD in artificial realizations of RR time series with 300, 500, and 900 samples. The RR time series are obtained by generating white uniform noise with a nominal standard deviation of $45 \mathrm{~ms}$ and adding an offset of $1000 \mathrm{~ms}$ (nominal $\mathrm{RR}_{\text {mean }}$ ). For each number of samples, 1000 realizations have been obtained. In each realization, the $\mathrm{RR}_{\text {mean }}, \mathrm{SDRR}$, and r-msDD have been calculated taking into account all the available samples. Their uncertainties and expanded uncertainties (95\% confidence level) have been computed by M1 and M2. The bias, estimated for each realization as the difference between each index computed with all the available samples or computed as the mean of the partial indices, has been also computed. At the end, the Monte Carlo uncertainty for each index has been computed as the standard deviation of the 1000 computed indices $\left(u_{\mathrm{MC}}\right)$. Moreover, the expanded uncertainty has been obtained as half the difference between the $97.5 \%$ and $2.5 \%$ percentiles $\left(U_{\mathrm{MC}}\right)$. For the uncertainties computed via M1 or M2, the mean \pm standard deviation was obtained.

2) Realizations From a First Order Autoregressive Model: An easy way to obtain nonindependent time series from a white noise generator is to use the independent samples as the input of a first order autoregressive model. Such approach has been employed in order to view the performance of M1 and M2 in correlated time series. The input of the model is white Gaussian noise $(w(n))$ and the output is defined as

$$
\operatorname{RR}(n)=\gamma \cdot \operatorname{RR}(n-1)+w(n) .
$$

The standard deviation of the input noise has been chosen in order to provide a RR time series with SDRR approximately equal to $45 \mathrm{~ms}$. We have tested seven different autoregressive models $(\gamma=0.7,0.5$, $0.3,0.0,-0.3,-0.5$ or -0.7$)$. For each model, 1000 realizations have been obtained with $N=300$ samples and a similar approach to the Monte Carlo simulation with independent samples has been followed.

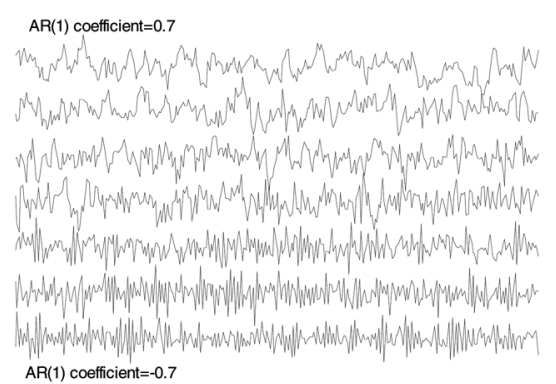

Fig. 3. Examples of realizations of nonindependent time series from a first order autoregressive model. The autoregressive coefficient decreases from the upper trace $(0.7)$ to the lower trace $(-0.7)$.

Fig. 3 shows a realization for each autoregressive model. At the output of the model an offset of $1000 \mathrm{~ms}$ has been added.

3) The Uncertainty of $R R_{\text {Mean }}, S D R R$, and r-msDD in Healthy Subjects: In the Normal Sinus Rhythm Database [11], that can be downloaded from www.physionet.org, 10236 RR time series, each with 300 intervals and without artifacts, where identified. For each time series, the $\mathrm{RR}_{\text {mean }}$, SDRR, and $\mathrm{r}$-msDD where computed with all the available samples and the uncertainty and expanded uncertainty (95\% confidence) where estimated by M2. This study provides margins of variation of the indices as well as their uncertainties in healthy subjects.

4) A Comparison of Indices: the Effect of Breathing Pattern On $R R_{\text {Mean }}, S D R R$, and r-msDD: For this example we say that two indices are statistically different (with a confidence greater than $\mathrm{p}$ ) if the two CIs obtained for the same confidence level $p$ does not overlap. Note that this test does not consider normality of the indices. Let us suppose two CIs with the same level of confidence for an index $I$

$$
\left.\begin{array}{l}
\text { Int }_{1}=I_{1} \pm U\left(I_{1}\right) \\
\text { Int }_{2}=I_{2} \pm U\left(I_{2}\right)
\end{array}\right\} \quad I_{1} \leq I_{2} .
$$

We define the interception value (IV) and the difference of indices (DI) as

$$
\begin{aligned}
& \mathrm{IV}=I_{2}-U\left(I_{2}\right)-I_{1}-U\left(I_{1}\right) \\
& \mathrm{DI}=I_{2}-I_{1} .
\end{aligned}
$$

If IV $>0$, the indices are different in a statistical sense (with a probability at least the confidence level). Moreover, if $I V \cong D I$, the effect of the expanded uncertainties is negligible so the affirmation that IV $>0$ implies that both indices are different is stronger. A quality factor $(Q)$ is defined as

$$
Q=\frac{1}{1-\ln \left(\frac{\mathrm{IV}}{\mathrm{DI}}\right)}
$$

$Q$ can take values between 0 and 1 and the greater its value, the stronger the affirmation of difference of indices is.

This test has been employed in $15 \mathrm{RR}$ time series when subjects were breathing at will (FB group) and in 15 RR time series when the same subjects were periodically breathing with breathing frequency between $0.15 \mathrm{~Hz}$ and $0.30 \mathrm{~Hz}$ (PB group). $\mathrm{FB}_{\mathrm{nn}}$ and $\mathrm{PB}_{\mathrm{nn}}$ recordings correspond to the same subject ( $\mathrm{n} n$ ranging from 01 to 15). Each time series corresponds to a measuring interval of $5 \mathrm{~min}$. More indications on the experimental setup can be found in [12]. The objective is to test for each subject ( 1 to 15 ) if $R_{\text {mean }}$, SDRR, and $r-m s D D$ have changed from free breathing (FB) to periodic breathing (PB). The CIs have been obtained for a $95 \%$ confidence level with M2 and each index has been estimated using all the available samples. 
TABLE II

RESUlts For the SimUlation With ARTIFICIAL RR Time SERIES With WHITE UNIFORM NOISE. FOR THE ESTIMATION OF THE BIAS $(b)$, STANDARD $(u)$, AND EXPANDED $(U)$ UnCERTAINTIES With THE M1 OR M2, THE MEAN AND STANDARD DEVIATION ARE REPORTED

\begin{tabular}{|c|c|c|c|c|}
\hline \multicolumn{2}{|c|}{ Parameter } & \multirow{2}{*}{$\begin{array}{c}N=300 \\
999.8\end{array}$} & \multirow{2}{*}{$\begin{array}{l}N=500 \\
1000.1\end{array}$} & \multirow{2}{*}{$\begin{array}{l}N=900 \\
1000.1\end{array}$} \\
\hline \multirow{9}{*}{ 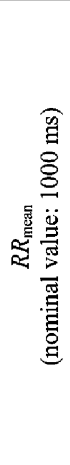 } & $\begin{array}{c}\overline{R R_{\text {mean }}} \\
\text { (ms) }\end{array}$ & & & \\
\hline & $\mathrm{b}_{\mathrm{M} 1}(\mathrm{~ms})$ & $0.02 \pm 0.55$ & $0.00 \pm 0.51$ & $0.00 \pm 0.37$ \\
\hline & $\mathrm{b}_{\mathrm{M} 2}(\mathrm{~ms})$ & $0.02 \pm 0.42$ & $0.00 \pm 0.36$ & $0.00 \pm 0.26$ \\
\hline & $\mathrm{u}_{\mathrm{MC}}(\mathrm{ms})$ & 2.67 & 2.10 & 1.50 \\
\hline & $\mathrm{u}_{\mathrm{MI}}(\mathrm{ms})$ & $2.64 \pm 0.42$ & $2.06 \pm 0.29$ & $1.55 \pm 0.18$ \\
\hline & $\mathrm{u}_{\mathrm{M} 2}(\mathrm{~ms})$ & $2.64 \pm 0.36$ & $2.05 \pm 0.25$ & $1.55 \pm 0.15$ \\
\hline & $\mathrm{U}_{\mathrm{MC}}(\mathrm{ms})$ & 5.07 & 3.98 & 2.91 \\
\hline & $\mathrm{U}_{\mathrm{M} 1}(\mathrm{~ms})$ & $5.53 \pm 0.88$ & $4.23 \pm 0.62$ & $3.13 \pm 0.39$ \\
\hline & $\mathrm{U}_{\mathrm{M} 2}(\mathrm{~ms})$ & $5.27 \pm 0.72$ & $4.10 \pm 0.49$ & $3.10 \pm 0.30$ \\
\hline \multirow{9}{*}{ 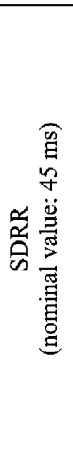 } & $\begin{array}{c}S D R R \\
(\mathrm{~ms})\end{array}$ & 44.97 & 45.03 & 44.99 \\
\hline & $\mathrm{b}_{\mathrm{M} 1}(\mathrm{~ms})$ & $0.46 \pm 0.65$ & $0.42 \pm 0.50$ & $0.40 \pm 0.39$ \\
\hline & $\mathrm{b}_{\mathrm{M} 2}(\mathrm{~ms})$ & $0.41 \pm 0.49$ & $0.39 \pm 0.36$ & $0.39 \pm 0.28$ \\
\hline & $\mathrm{u}_{\mathrm{MC}}(\mathrm{ms})$ & 1.19 & 0.87 & 0.67 \\
\hline & $\mathrm{u}_{\mathrm{Ml}}(\mathrm{ms})$ & $1.31 \pm 0.23$ & $1.03 \pm 0.15$ & $0.75 \pm 0.09$ \\
\hline & $\mathrm{u}_{\mathrm{M} 2}(\mathrm{~ms})$ & $1.32 \pm 0.18$ & $1.02 \pm 0.11$ & $0.76 \pm 0.06$ \\
\hline & $\mathrm{U}_{\mathrm{MC}}(\mathrm{ms})$ & 2.41 & 1.76 & 1.32 \\
\hline & $\mathrm{U}_{\mathrm{Ml}}(\mathrm{ms})$ & $2.74 \pm 0.47$ & $2.10 \pm 0.30$ & $1.52 \pm 0.17$ \\
\hline & $\mathrm{U}_{\mathrm{M} 2}(\mathrm{~ms})$ & $2.63 \pm 0.37$ & $2.04 \pm 0.23$ & $1.51 \pm 0.13$ \\
\hline \multirow{9}{*}{ 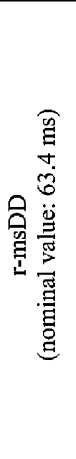 } & $r-\underset{(\mathrm{ms})}{m S D D}$ & 63.67 & 63.57 & 63.66 \\
\hline & $\mathrm{b}_{\mathrm{M} 1}(\mathrm{~ms})$ & $-1.06 \pm 0.82$ & $-1.03 \pm 0.66$ & $-1.00 \pm 0.60$ \\
\hline & $\mathrm{b}_{\mathrm{M} 2}(\mathrm{~ms})$ & $-1.01 \pm 0.56$ & $-1.01 \pm 0.46$ & $-0.96 \pm 0.40$ \\
\hline & $\mathrm{u}_{\mathrm{MC}}(\mathrm{ms})$ & 2.49 & 1.89 & 1.45 \\
\hline & $\mathrm{u}_{\mathrm{Ml}}(\mathrm{ms})$ & $2.62 \pm 0.43$ & $2.03 \pm 0.29$ & $1.52 \pm 0.18$ \\
\hline & $\mathrm{u}_{\mathrm{M} 2}(\mathrm{~ms})$ & $2.60 \pm 0.39$ & $2.02 \pm 0.24$ & $1.52 \pm 0.15$ \\
\hline & $\mathrm{U}_{\mathrm{MC}}(\mathrm{ms})$ & 4.75 & 3.50 & 2.81 \\
\hline & $\mathrm{U}_{\mathrm{M} 1}(\mathrm{~ms})$ & $5.50 \pm 0.93$ & $4.17 \pm 0.64$ & $3.08 \pm 0.38$ \\
\hline & $\mathrm{U}_{\mathrm{M} 2}(\mathrm{~ms})$ & $5.20 \pm 0.78$ & $4.04 \pm 0.49$ & $3.03 \pm 0.30$ \\
\hline
\end{tabular}

\section{RESULTS}

Table II shows the results for the simulation with uniform white noise. For the mean RR, M1, M2, and Monte Carlo provide similar results for the standard uncertainty. Nevertheless, the expanded uncertainty is a little overestimated with M1 and M2. Regarding the uncertainties for SDRR and r-msDD, both standard and expanded uncertainties with M1 and M2 are overestimated by a $10 \%-20 \%$. The bias in $\mathrm{M} 1$ and M2 is very low for the mean RR, approximately a $1 \%$ for the SDRR and lower than $2 \%$ for the r-msDD.

Fig. 4 shows the results for the simulation with autoregressive models. In the figure, only mean values of the uncertainties and biases are displayed. The more correlated the samples are (coefficient more positive), the higher the uncertainty on the mean and the lower the uncertainty on the r-msDD. The uncertainty for SDRR has a minimum when the samples are independent. In highly correlated time series, M1 and M2 have high biases in the estimation of SDRR (nearly a $17 \%$ for an AR coefficient of 0.7 ) while the standard uncertainty of
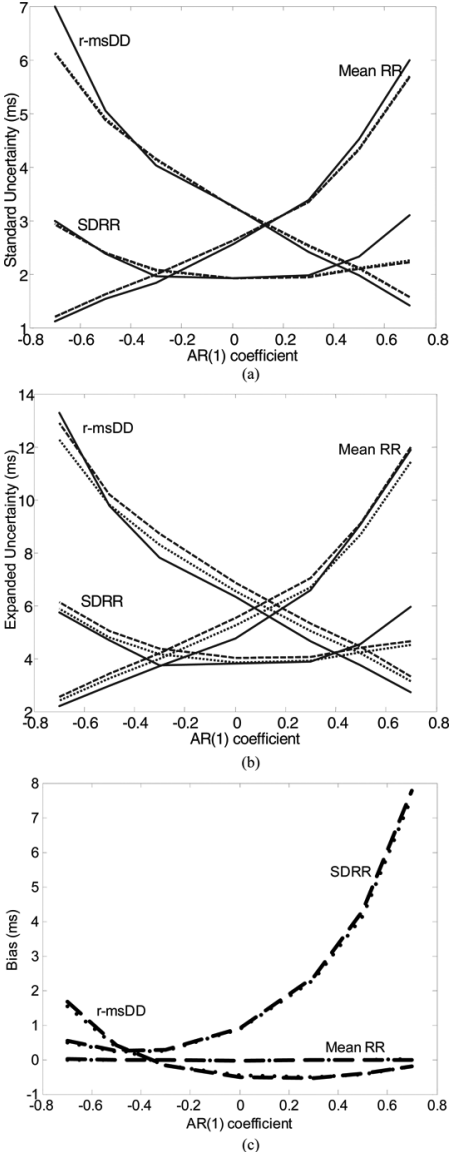

Fig. 4. (a) Standard and (b) expanded uncertainty of the three indices obtained by Monte Carlo simulation and M1 and M2 methods using simulated nonindependent time series (first order autoregressive model) plotted against the autoregressive coefficient. (c) Mean value of the bias. The solid line is the result of the uncertainty computed by the Monte Carlo method, the dashed and dotted line correspond to the mean values of uncertainty or bias obtained via M1 and M2 respectively.

this index is underestimated. With an AR coefficient of -0.7 the same problem arises in the estimation of the standard uncertainty of r-msDD although the bias with M1 and M2 is lower.

Fig. 5 shows the results for the relative standard uncertainty (standard uncertainty normalized by the value of the index) in the three indices computed for the normal sinus rhythm database. As seen, the uncertainty for the mean is low but for the SDRR and r-msDD it can be higher than the $10 \%$ of the index. By averaging the results, the mean relative standard uncertainty for the mean is $1.08 \%$ (Range: $0.1 \%-9.0 \%$ ), for the SDRR is $6.27 \%$ (Range: $0.81 \%-22.8 \%$ ), and for the r-msDD is $6.98 \%$ (Range: $2.11 \%-24.4 \%$ ). Note that these uncertainties depend on $N$. In this case $N=300$, but with the same dynamics of the time series, a reduction of the number of available samples implies an increase in uncertainty.

Table III shows the results for the 15 comparisons of indices [FB versus $\mathrm{PB}]$. For each pair of RR time series and index, the two indices, the interception value, the difference of indices, and the quality factor are reported. As seen in Table III, only subjects 3, 4, and 5 have significant changes in the three indices while subjects 7,10 , and 11 have not noticeable changes in neither index. Nine subjects experience a significant change in $\mathrm{RR}_{\text {mean }}$, four subjects in SDRR and seven subjects in r-msDD. Fig. 6 shows the time series and results (indices \pm standard uncertainties) for subjects 5 and 10 . As seen, the RR time series of subject 5 is very different when breathing periodically respect to breathing 


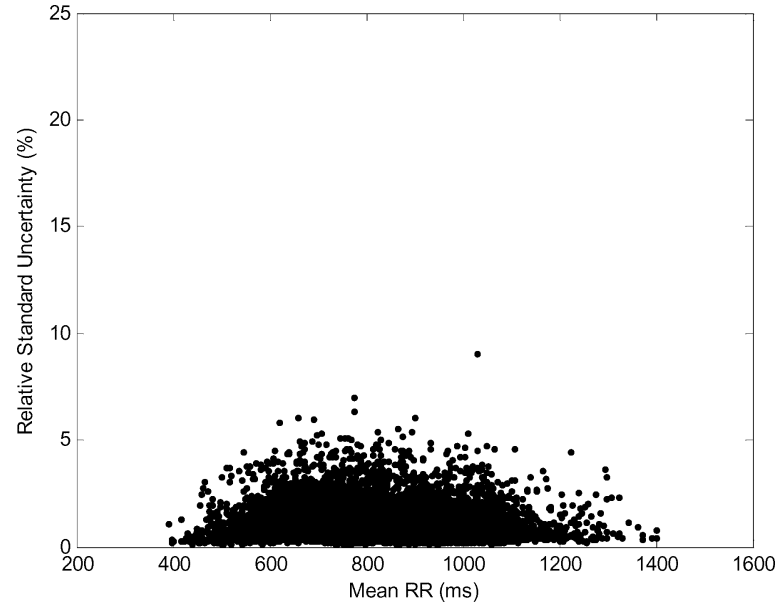

(a)

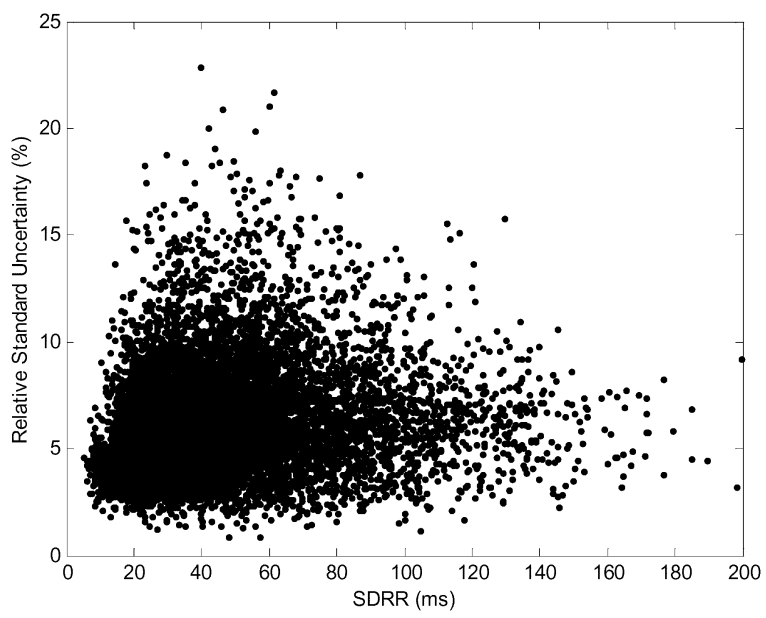

(b)

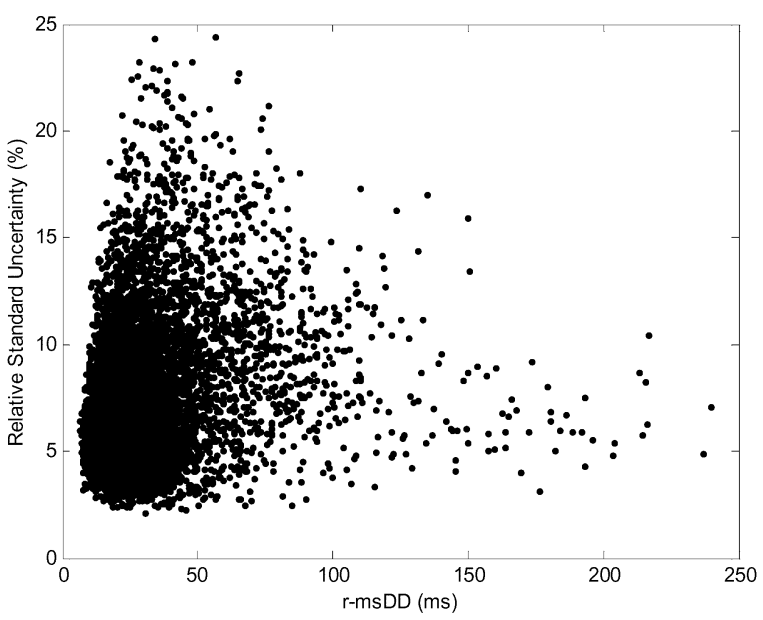

(c)

Fig. 5. Relative standard uncertainty versus Time domain indices for $10236 \mathrm{RR}$ time series of the normal sinus rhythm database with 300 samples each. Results for (a) RR mean, (b) SDRR, and (c) r-msDD.

at will. Nevertheless, in subject 10 , there is no remarkable change in the studied indices.

\section{DISCUSSION}

The described methodology provides a way to estimate the uncertainty in indices obtained from a quantification of RR time series. This method is not suitable (or applicable in short recordings) for any index of HRV. For example, spectral indices require a higher number of samples due to spectral resolution issues. A partial spectrum with 10-30 samples will be very inaccurate. Another example is the estimation of the Hurst exponent by detrended fluctuation analysis [13]. The method requires a high number of samples and, in this situation, the assumption of stationarity does not hold. In this paper, we have dealt with RR time series with $N=300$ (the studies in the normal sinus rhythm database) or $5 \mathrm{~min}$ long (the experiment of free and $\mathrm{PB}$ ). The study of the uncertainty of indices from 24-h recordings is out of the scope of this paper because the RR time series is not stationary. Moreover, the assumption of both M1 and M2 is that the mean of partial indices will be approximately equal to the index computed in the overall recording. If not, the index is not consistent and the methodology cannot be applied.

As seen in Fig. 4, M1 and M2 provide underestimated standard uncertainties of SDRR and r-msDD when the bias is high. The bias can be estimated for each RR time series by comparing the mean of partial indices and the index computed with all the available samples. This bias can be employed as another indicator of the quality of the estimation of the uncertainties. A further study on the bias can reveal ways to correct the uncertainties in order to avoid this underestimation. Nevertheless, the need for the correction of the underestimation of the uncertainties must be in accordance with the accuracy desired for a particular application of the index. As an example, if the difference of two indices is higher than ten times the higher standard uncertainty of both indices obtained by M1 or M2, it is useless to refine the estimation of the uncertainties because the result of the test will be that both indices are statistically different.

This paper provides also some guidance to the computation of the CI. We rely on the t-Student distribution to obtain such intervals although the normality of the partial indices is not tested. Nevertheless, the violation of the normal distribution assumption does not significantly compromise the results of the test if the distribution has not a severe skew [14]. The 95th percentile of the absolute sample skewness of the partial indices involved in the M1 computation for the normal sinus rhythm database is 1.6 for the mean RR, 2.3 for the SDRR, and 2.2 for the $\mathrm{r}-\mathrm{msDD}$ while the mean value of the absolute sample skewness is 0.59 for the mean RR, 0.98 for the SDRR, and 0.76 for the $\mathrm{r}$-msDD. So, we can conclude that the distribution of these partial indices have not a severe skew.

The strategy for the search of sets of independent indices is not complete. In the proposed approach we have supposed that once the first block ends at an RR sample, the next block starts just at the next sample. Of course, some samples can separate successive blocks. In the proposed method, each block has the same number of RR samples for simplicity and two methods (M1 and M2) have been suggested in order to combine the results for the whole set of independent partial indices. Both methods provide similar standard uncertainties that are close to the Monte Carlo simulations for independent and correlated time series. Nevertheless, the CIs are overestimated for independent data. Maybe another combination of sets can provide a better estimation of the expanded uncertainty.

The proposed method looks quite similar to the block bootstrap method to obtain the uncertainty of statistical estimators in time series [15]. Nevertheless, there are several important differences between them. The block bootstrap computes the index in several blocks with a certain length that not varies in the computation and has to been fixed according to an empirical criterion or by fitting the time series to a model. Moreover, there is no check of the independence of the obtained partial indices. An expression similar to (3) is employed in 
TABLE III

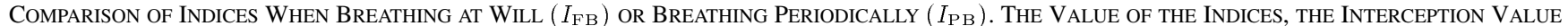
(IV), THE DIFFERENCE OF INDICES (DI) AND THE QUALITY FACTOR $(Q)$ IS REPORTED FOR THE 15 SUBJECTS AND FOR THE MEAN RR, SDRR, AND R-MSDD INDICES. WHEN IV IS NEGATIVE, $Q$ Is REPORTED AS N.S. BECAUSE THERE IS NO SigNIFICANT DIFFERENCE FOR THE INDEX

\begin{tabular}{|c|c|c|c|c|c|c|c|c|c|c|c|c|c|c|c|c|}
\hline \multicolumn{2}{|c|}{ \# subject $(\mathrm{nn})$} & 01 & 02 & 03 & 04 & 05 & 06 & 07 & 08 & 09 & 10 & 11 & 12 & 13 & 14 & 15 \\
\hline \multirow{5}{*}{ 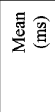 } & $I_{F B}(\mathrm{~ms})$ & 1137 & 1389 & 823.0 & 946.2 & 665.7 & 937.5 & 967.0 & 982.9 & 1063 & 1081 & 916.6 & 1018 & 764.7 & 1171 & 1114 \\
\hline & $I_{P B}(\mathrm{~ms})$ & 1193 & 1284 & 875.6 & 891.3 & 738.8 & 832.0 & 959.6 & 997.5 & 1037 & 1069 & 916.2 & 939.7 & 804.6 & 1075 & 1088 \\
\hline & $I V(\mathrm{~ms})$ & 9.91 & 42.7 & 19.0 & 24.6 & 57.0 & 79.1 & -30.8 & -40.1 & -10.5 & -32.1 & -44.1 & 14.89 & 19.5 & 66.0 & -6.53 \\
\hline & $D I(\mathrm{~ms})$ & 55.7 & 104.7 & 52.6 & 59.91 & 73.04 & 105.5 & 7.37 & 14.6 & 26.4 & 11.4 & 0.35 & 78.7 & 39.91 & 96.3 & 25.4 \\
\hline & $Q$ & 0.37 & 0.53 & 0.50 & 0.55 & 0.80 & 0.78 & n.s. & n.s. & n.s. & n.s. & n.s. & 0.38 & 0.58 & 0.73 & n.s. \\
\hline \multirow{5}{*}{ 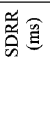 } & $I_{F B}(\mathrm{~ms})$ & 123.2 & 139.8 & 52.90 & 75.92 & 23.57 & 101.8 & 85.2 & 84.97 & 67.9 & 82.8 & 111.8 & 134.8 & 32.92 & 71.5 & 69.3 \\
\hline & $I_{P B}(\mathrm{~ms})$ & 136.2 & 148.8 & 80.15 & 100.4 & 59.45 & 87.73 & 84.4 & 96.01 & 99.5 & 95.7 & 109.7 & 137.7 & 38.68 & 68.9 & 62.3 \\
\hline & $I V(\mathrm{~ms})$ & -15.2 & -30.0 & 12.0 & 7.68 & 25.04 & -7.52 & -15.2 & -8.97 & 12.2 & -9.1 & -20.9 & -25.6 & -1.72 & -14.1 & -11.1 \\
\hline & $D I(\mathrm{~ms})$ & 13.0 & 9.04 & 27.2 & 24.4 & 35.88 & 14.10 & 0.85 & 11.05 & 31.6 & 12.9 & 2.09 & 2.86 & 5.76 & 2.54 & 6.97 \\
\hline & $Q$ & n.s. & n.s. & 0.55 & 0.46 & 0.74 & n.s. & n.s. & n.s. & 0.51 & n.s. & n.s. & n.s. & n.s. & n.s. & n.s. \\
\hline \multirow{5}{*}{ 会奥 } & $I_{F B}(\mathrm{~ms})$ & 139.6 & 150.1 & 33.99 & 68.83 & 16.27 & 100.1 & 62.2 & 52.05 & 66.2 & 80.1 & 86.2 & 114.3 & 22.60 & 83.7 & 55.7 \\
\hline & $I_{P B}(\mathrm{~ms})$ & 149.4 & 170.8 & 73.79 & 89.99 & 45.60 & 77.8 & 74.6 & 84.5 & 91.4 & 89.1 & 103.8 & 132.3 & 31.09 & 58.3 & 58.3 \\
\hline & $I V(\mathrm{~ms})$ & -21.2 & -20.9 & 21.96 & 3.73 & 19.5 & -4.14 & -3.13 & 14.5 & 7.58 & -9.91 & -9.20 & -16.8 & 1.48 & 4.15 & -13.9 \\
\hline & $D I(\mathrm{~ms})$ & 9.80 & 20.7 & 39.81 & 21.17 & 29.3 & 22.3 & 12.47 & 32.5 & 25.3 & 9.05 & 17.7 & 18.0 & 8.49 & 25.5 & 2.55 \\
\hline & $Q$ & n.s. & n.s. & 0.63 & 0.37 & 0.71 & n.s. & n.s. & 0.55 & 0.45 & n.s. & n.s. & n.s. & 0.36 & 0.36 & \begin{tabular}{|l|} 
n.s. \\
\end{tabular} \\
\hline
\end{tabular}

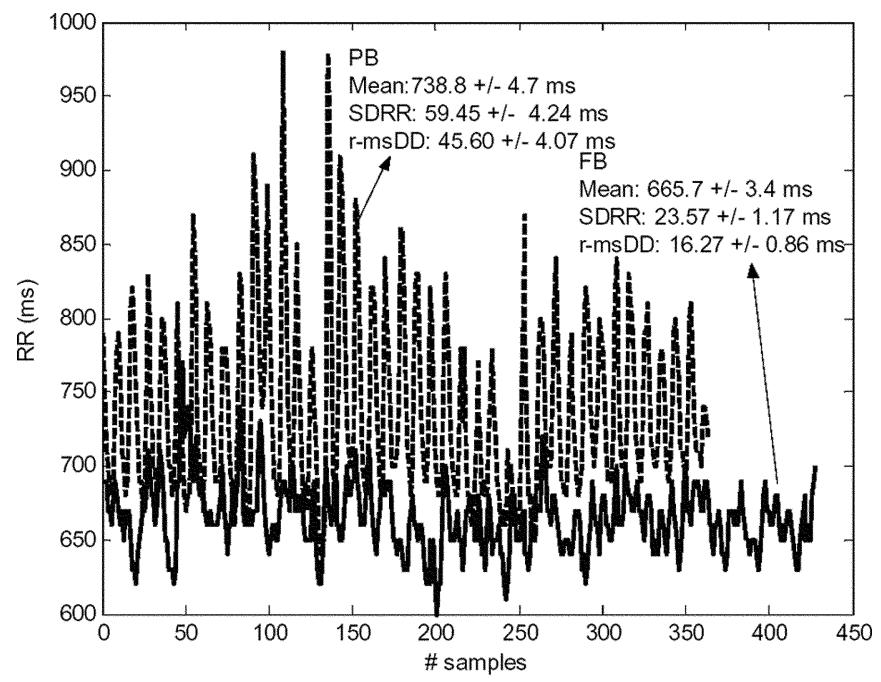

(a)

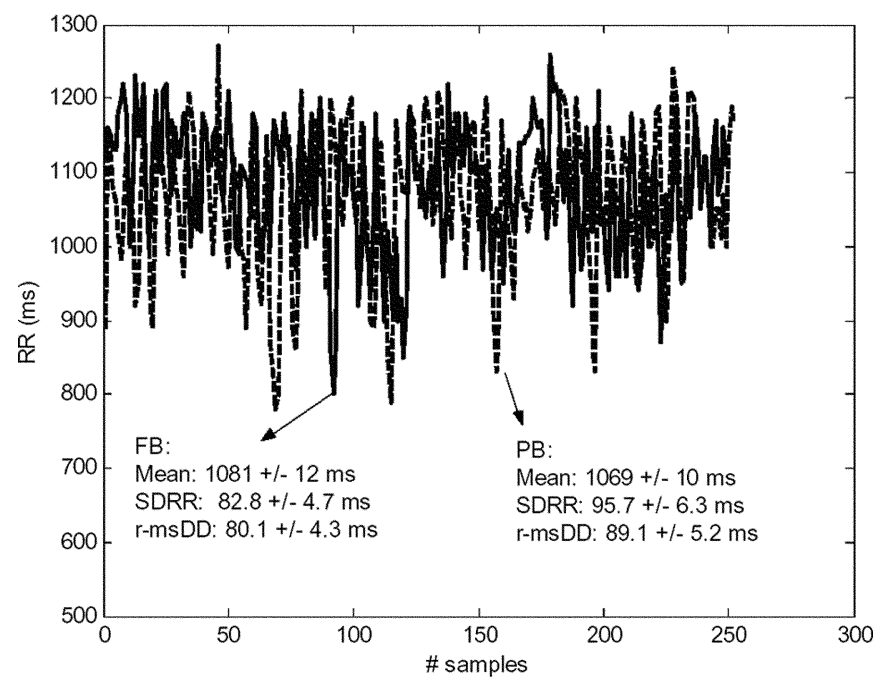

(b)

Fig. 6. Example of comparison of indices: The RR time series and indices \pm standard uncertainties for subjects (a) 05 and (b) 10 in the analysis of the effect of the breathing pattern are displayed. The solid line corresponds to the RR time series while FB and the dashed line corresponds to the RR time series while PB.

order to obtain the uncertainty. Moreover, the blocks can repeat some intervals. In our approach we test the independence of the partial indices and test different block lengths $(n)$. If an interval has been employed in the computation of a partial index, this interval can not be employed for the computation of another partial index. Moreover, $n$ changes in order to find sets that have the lowest IF.

Regarding M2, an incidence is reported if no set with IF lower than 1 has been found. In the case of periodic and FB no incidence has been reported (the measurements where obtained with a controlled experimental setup). The analysis of the normal sinus rhythm database is different because the RR time series are obtained from Holter monitoring. In this case, $24 \%$ of the RR time series reported incidence in the computation of the uncertainty of the mean, $3 \%$ in the computation of the uncertainty of the SDRR and 5\% in the computation of the r-msDD. Nevertheless, the uncertainties obtained when incidences are present do not differ from uncertainties obtained without incidences (the two groups, with and without incidence, form "clouds" in Fig. 5 that overlap and cannot be separated)

\section{CONCLUSION}

A methodology for estimating the uncertainty of some time domain indices of RR time series has been presented. For the uncertainty calculation, the time series must come from independent observations and its stationarity is necessary. In order to check the independence, a strategy for choosing independent sets of indices has been designed. Two different methods based on the value of an independence factor have been employed in order to assess the uncertainty of the indices. The methodology has been applied to artificial independent and dependent time series in order to test the methodology goodness. The same methodology has been applied to several records of Normal Sinus Rhythm Database obtaining the values of the indices uncertainties in healthy subjects. We have found that the SDRR and r-msDD uncertainties can be higher than the $10 \%$ of the index.

We have also presented a method to decide if two indices are statistically different, by using a quality factor $\mathrm{Q}$ calculated from the difference and CI of both indices. We have applied this methodology to the separation of indices of RR time series of the same subject when breathing at will and with PB. We have found that the changes in indices depend on the subject and on the index itself.

\section{REFERENCES}

[1] Task Force of the European Society of Cardiology and the North American Society of Pacing and Electrophysiology, "Heart rate variability, standards of measurement, physiological interpretation, and clinical use," Eur. Heart., vol. J17, pp. 354-381, 1996.

[2] J. Nolan et al., "Prospective study of heart rate variability and mortality in chronic heart failure: Results of the united kingdom failure evaluation and assessment of risk trial (UK-Heart)," Circulation, vol. 98, pp. 1510-1516, 1998. 
[3] H. V. Huikuri et al., "Prediction of sudden cardiac death after myocardial infarction in the beta-blocking era," J. Am. Coll. Cardiol., vol. 42, pp. 652-658, 2003.

[4] H. Tsuji et al., "Impact of reduced heart rate variability on risk for cardiac events: The framingham heart study," Circulation, vol. 94, pp. 2850-2855, 96.

[5] JT Bigger et al., "The ability of several short-term measures of RR variability to predict mortality after myocardial infarction," Circulation, vol. 88, pp. 927-934, 1993.

[6] E. R. Migliaro, R. Canetti, P. Contreras, and M. Hakas, "Heart rate variability: Short term studies are as useful as holter to differentiate diabetic patients form healthy subjects," Ann. Noninvasive Electrocardiol., vol. 8, pp. 313-320, 2003.

[7] A. E. Aubert, B. Seps, and F. Beckers, "Heart rate variability in athletes," Sports Med., vol. 33, pp. 889-919, 2003.

[8] R. H. Wood, W. A. Wood, M. Welsch, and P. Avenal, "Physical activity, mental stress, and short-term heart rate variability in patients with ischemic heart disease," J. Cardiopulmonary Rehabil., vol. 18, pp. 271-276, 1998.

[9] Guide to the Expression of Uncertainty in Measurement International Organization for Standardization, Switzerland, 1995.

[10] J. S. Bendat and A. G. Piersol, Random Data, Analysis and Measurement Procedure, 2nd ed. New York: Wiley, 1986.

[11] A. L. Goldberger et al., "PhysioBank, physiotoolkit, and physionet: Components of a new research resource for complex physiologic signals," Circulation, vol. 101, pp. e215-e220, 2000.

[12] M. A. García-González, C. Vázquez-Seisdedos, and R. Pallàs-Areny, "Variations in breathing patterns influence HRV spectral analysis," Physiol. Meas., vol. 21, pp. 417-423, 2000.

[13] C. K. Peng et al., "Long-range anticorrelations and non-Gaussian behaviour of the heartbeat," Phys. Rev. Lett., vol. 70, pp. 1342-1346, 2000.

[14] J. M. Stonehouse and G. J. Forrester, "Robustness of the t and U tests under combined assumption violations," J. Appl. Statist., vol. 25, pp. 63-75, 1998.

[15] H. R. Kunsch, "The jackknife and the bootstrap for general stationary observations," Ann. Statist., vol. 17, pp. 1217-1241, 1989.

\section{Selective Activation of Distant Nerve by Surface Electrode Array}

Hirokazu Takahashi*, Masayuki Nakao, and Kimitaka Kaga

\begin{abstract}
Neural prostheses for restoring lost functions can benefit from selective activation of nerves with limited number and density of electrodes. Here, we show by simulations and animal experiments that multipoint simultaneous stimulation with a surface electrode array can selectively activate nerves in a bundle at a desired location in between the array or at a desired depth, which are referred to as lateral or depth-wise gating stimulation, respectively. The stimulation broadly generates action potentials with cathodic source electrodes, and simultaneously blocks unnecessary propagation with downstream anodic gate electrodes. In general, stimulation with a small diameter electrode can affect a nearly hemispherical region, while a large electrode is effective at a more vertically compressed region, i.e., a surface of nerve bundle. The gating stimulation takes advantage of the size effects by utilizing an asymmetrical electrode array. The array of the lateral gating stimulation is designed to have four electrodes; a pair of large source electrodes and a pair of small gate electrodes. The depth-wise gating stimulation array consists of two electrodes; a large gate and small source electrodes. The simulation first demonstrated that appropriate combination of currents at the source and gate electrodes can change recruitment patterns of nerves with lateral or depth-wise selectivity as desired. We then applied the lateral gating stimulation on the rat spinal cords and obtained a preliminary support for the feasibility.
\end{abstract}

Index Terms-Extracellular stimulation, functional electrical stimulation (FES), neural prosthesis, , rat, spinal cord.

\section{INTRODUCTION}

Electrical stimulation with an implanted electrode array can restore some sensory and motor functions [1]-[5]. To increase the prosthetic functions, a large number of nerves must be selectively activated, which in turn may cause complicated implantation and maintenance of a large number of electrodes. Conventional prostheses exclusively activate nerves confined around the electrode, and thereby the performance largely depends on the location and configuration of the electrode array [6]-[11]. In these neural prostheses, therefore, precise and selective nerve activation with limited number and density of electrodes would be ideal. In this paper, we propose a novel stimulation technique named multipoint "gating stimulation," which utilizes the combined effects of anodic and cathodic stimulation, and allows a surface electrode array to selectively activate nerves at a desired location in between the array or at a desired depth beneath the array.

Previous work empirically investigated how axons reacted to extracellular stimuli [8], and mathematically modeled the reaction [9]-[11].

Manuscript received September 19, 2005; revised August 26, 2006. Astersik iindicates corresponding author.

*H. Takahashi is with the Research Center for Advanced Science and Technology, The University of Tokyo, 4-6-1 Komaba, Meguro-ku, Tokyo 153-8904, Japan. the Department of Mechano-informatics, Graduate School of Information Science and Technology, The University of Tokyo 7-3-1 Hongo, Bunkyo-ku, Tokyo 113-8656, Japan. and the Department of Engineering Synthesis, Graduate School of Engineering, The University of Tokyo 7-3-1 Hongo, Bunkyo-ku, Tokyo 113-8656, Japan. (e-mail: takahashi@i.u-tokyo.ac.jp).

M. Nakao is with the Department of Engineering Synthesis, Graduate School of Engineering, The University of Tokyo, Tokyo 113-8656, Japan.

K. Kaga is with the Department of Otolaryngology, and Head and Neck Surgery, Graduate School of Medicine, The University of Tokyo, Tokyo 113-8655, Japan.

Digital Object Identifier 10.1109/TBME.2006.890509 